\title{
TRANSFORMATIONS OF MULTIPLE FOURIER SERIES
}

\author{
L. B. HEDGE
}

1. Introduction. The object of the present paper is the study and characterization of certain classes of factor sequence transformations of multiple Fourier series. A recent moment problem solution ${ }^{1}$ by the author and a scheme of summation of multiple Fourier series developed by Bochner ${ }^{2}$ are used in the study. The results include and extend known results for single Fourier series.

2. Definitions and notation. Let $n$ be a positive integer, fixed but arbitrary. $R^{n}$ will denote the euclidean $n$-space. $(x),(y)$, and so on will denote $\left(x_{1}, x_{2}, \cdots, x_{n}\right),\left(y_{1}, y_{2}, \cdots, y_{n}\right)$, and so on, points of $R^{n}$. $\nu, \tau, j, k, s$ will be used for non-negative integers, and $(\nu),(\tau)$, and so on will be used for $\left(\nu_{1}, \nu_{2}, \cdots, \nu_{n}\right),\left(\tau_{1}, \tau_{2}, \cdots, \tau_{n}\right)$, lattice points of $R^{n}$. (0) will mean $(0,0, \cdots, 0)$, and $(x)=(y)$ will mean $x_{j}=y_{j}$, $j=1,2, \cdots, n$. $(k \cdot x)$ will stand for the number $k_{1} x_{1}+k_{2} x_{2}+\cdots+k_{n} x_{n}$, $|x|$ for the number $\left(x_{1}^{2}+x_{2}^{2}+\cdots+x_{n}^{2}\right)^{1 / 2} . A, I$, and $\lambda$ will be used for functions defined on the lattice points of $R^{n}$, and $I$ will be the characteristic function of the lattice points of $R^{n}$. $E$ will be the set $E_{(x)}\left(-\pi \leqq x_{j}<\pi, j=1,2, \cdots, n\right) . R$ and $t$ will be used for real numbers. $(x+y)$ will stand for $\left(x_{1}+y_{1}, x_{2}+y_{2}, \cdots, x_{n}+y_{n}\right)$, and $B(n)$ for a real constant depending only on $n$. $\Phi$ will be used for a function $U^{*}$ of bounded variation in the sense of Saks, and if $\Phi(H)$ $=\Phi_{1}(H)+\Phi_{2}(H)$ for any Borel set $H$ with $\Phi_{1}(H) \geqq 0 \geqq \Phi_{2}(H)$ we will write $\int_{H} f(x)|d \Phi(E)|$ for $\int_{H} f(x) d \Phi_{1}(E)-\int_{H} f(x) d \Phi_{2}(E)$. When $\Phi$ is the Lebesgue measure function we will write $\int_{H} f(x) d x$ for

$$
\int_{H} f(x) d \Phi(E) .
$$

We will write $f \in L$ to indicate that $\int_{E} f(x) d x$ exists, and $f \in C$ to indicate that $f$ is continuous on $\bar{E}$ and $f(x)=f(x+y)$ for all combinations of $y_{j}=0$ or $2 \pi, j=1,2, \cdots, n$. A function $f$ defined over $E$ will be defined over $R_{n}$ by the extension $f(x)=f(x+y)$ with $y_{j}=0$ or $2 \pi$, $j=1,2, \cdots, n$.

Let

Presented to the Society, December 30,1940; received by the editors May 17, 1942.

${ }^{1}$ L. B. Hedge, Moment problem for a bounded region, Bull. Amer. Math. Soc. vol. 47 (1941) pp. 282-285. Referred to later as MP.

${ }^{2} \mathrm{~S}$. Bochner, Summation of multiple Fourier series by spherical means, Trans. Amer. Math. Soc. vol. 40 (1936) pp. 175-207. 


$$
\Im(\lambda, A, x) \simeq \sum_{(k)} \lambda(k) A(k) e^{i(k \cdot x)}
$$

and

$$
\Im_{\nu}(\lambda, A, x)=\sum_{|k|^{2} \leqq \nu} \lambda(k) A(k) e^{i(k \cdot x)}
$$

If

$$
\lambda(k) A(k)=(2 \pi)^{-n} \int_{E} f(x) e^{-i(k \cdot x)} d x
$$

or

$$
\lambda(k) A(k)=(2 \pi)^{-n} \int_{E} e^{-i(k \cdot x)} d \Phi(E), \quad \int_{E}|d \Phi(E)|<\infty,
$$

we will write $\subseteq(f, x)$ or $\subseteq(d \Phi, x)$, respectively, for the left side of equation (1), and similarly alter equations (2) and (5).

We write

$$
S_{R}(\lambda, A, x)=\sum_{(k)} \Psi(|k| / R) \lambda(k) A(k) e^{i(k \cdot x)}
$$

where

$$
\Psi(t)=e^{-t^{2}}
$$

for the Bochner-Gauss ${ }^{2}$ spherical means of the sequence (2), and

$$
K_{R}(u)=\sum_{(k)} \Psi\left(\frac{|K|}{R}\right) e^{-i(k \cdot u)}=S_{R}(I, I, u)
$$

for the corresponding kernel.

3. Spherical summation. We proceed to some modification (largely notational) of the Bochner summation theory. The transformation which takes (2) into (5) is given by the matrix $T:\left\|a_{R, \nu}\right\|$, where $a_{R, \nu}=\Psi\left(R_{\nu} / R\right)-\Psi\left(R_{\nu+1} / R\right)$, and $\left\{R_{\nu}\right\}$ is a subsequence of $\left\{0,1,2^{1 / 2}\right.$, $\left.3^{1 / 2}, 4^{1 / 2}, \cdots, k^{1 / 2}, \cdots\right\}$. We have at once

$$
a_{R, \nu} \geqq 0, \quad \lim _{R \rightarrow \infty} a_{R, \nu}=0, \quad \sum_{\nu=0}^{\infty} a_{R, \nu}=1,
$$

whence $T$ is a regular Toeplitz transformation. ${ }^{3}$

${ }^{3}$ See, for example, A. Zygmund, Trigonometrical series, Monografje Matematyczne, vol. 5, Warsaw, 1935, pp. 79-86. That $R$ is a continuous variable is unimportant. 
The following properties of the summation scheme follow immediately from Bochner's work:

$$
\begin{aligned}
& 0 \leqq K_{R}(u) \leqq M_{R}<\infty, \\
& S_{R}(f, x)=B(n) \int_{E} f(u) K_{R}(u-x) d u, \quad \text { if } f \in L,
\end{aligned}
$$

and,

$$
S_{R}(f, x) \rightarrow f(x)
$$

at every point $(x)$ of continuity of $f$, and uniformly on $E$ if $f \in C$.

4. Classes of multiple Fourier series. The theorem of MP may now be given in the following form:

Theorem. In order that $\subseteq(\lambda, A, x):=\subseteq(d \Phi, x)$ with

$$
\text { 1. } \int_{E}|d \Phi| \leqq M, \quad \text { or } \quad 2 . \quad \Phi \geqq 0,
$$

it is necessary and sufficient that

$$
\text { 1. } \int_{E}\left|S_{R}(\lambda, A, x)\right| d x \leqq M, \quad \text { or } \quad \text { 2. } \quad S_{R}(\lambda, A, x) \geqq 0,
$$

and in order that $\mathfrak{S}(\lambda, A, x)=\subseteq(f, x)$ with

$$
\text { 3. } f \in L, \quad \text { or } \quad \text { 4. }|f| \leqq M \text {, or } 5 \text {. } f \in C \text {, }
$$

it is necessary and sufficient that $3 .\left\{S_{R}(\lambda, A, x)\right\}$ converge in the mean with exponent 1 , or $4 .\left|S_{R}(\lambda, A, x)\right| \leqq M$, or $5 .\left\{S_{R}(\lambda, A, x)\right\}$ converge uniformly in $B$.

We shall write $\mathfrak{S}(\lambda, A, x) \in S$ to indicate that $\mathfrak{S}(\lambda, A, x)=\mathfrak{S}(d \Phi, x)$ with $\int_{E}|d \Phi| \leqq M$, and $\Im(\lambda, A, x) \in L, M$, or $C$, if $\Im(\lambda, A, x)=\Im(f, x)$ with $f \in L, f \in M$ or $f \in C$ respectively. We will write $\lambda \in(P, Q)$ to indicate that $\mathfrak{S}(I, A, x) \in P$ implies $\subseteq(\lambda, A, x) \in Q$.

5. Transformations. We begin with the following lemma.

Lemma 1. $\subseteq(I, I, x) \in S$.

Proof. Let $f \in C$. Since $S_{R}(I, I, x)=K_{R}(x)$, we have

$$
S_{R}(f, x)=B(n) \int_{E} f(u) S_{R}(I, I, u-x) d u,
$$

but $f$ is continuous, the left-hand member converges uniformly to $f$, 
and for any sequence of values of $R,\left\{\left|S_{R}(f, x)\right|\right\}$ is bounded uniformly. $\left\{S_{R}(f, x)\right\}$ is a sequence of linear operations whose norms are

$$
\int_{E}\left|S_{R}(I, I, x)\right| d x
$$

and by a simple corollary of a theorem of Banach and Steinhaus ${ }^{4}$ these must be uniformly bounded, or $\subseteq(I, I, x) \in S$.

We now have this theorem.

Theorem 1. The transformation classes $(S, S),(M, M),(L, L)$, and $(C, C)$ are identical, and $\lambda$ belongs to each of them if and only if $\Im(\lambda, I, x) \in S$, or

$$
\int_{E}\left|S_{R}(\lambda, I, x)\right| d x \leqq M .
$$

(A) $\lambda \in(S, S) \rightarrow(10)$. This follows immediately from Lemma 1 .

(B) $(10) \rightarrow \lambda \in(S, S)$. From (5) we have

$$
\begin{aligned}
S_{R}(\lambda, A, x) & =\sum_{(k)} \Psi\left(\frac{|K|}{R}\right) \lambda(k) A(k) e^{i(k \cdot x)} \\
& =\sum_{(k)} \Psi\left(\frac{|K|}{R}\right) \lambda(k)(2 \pi)^{-n} \int_{E(u)} e^{i(k \cdot x-u)} d \Phi(E)^{5} \\
& =(2 \pi)^{-n} \int_{E(u)} S_{R}(\lambda, I, x-u) f(u) d \Phi(E),
\end{aligned}
$$

whence

$$
\int_{E}\left|S_{R}(\lambda, A, x)\right| d x \leqq(2 \pi)^{-n} \cdot M \cdot \int_{E}|d \Phi| .
$$

(C) $\lambda \in(M, M) \rightarrow(10)$ and $\lambda \in(C, C) \rightarrow(10)$.

We have immediately in both cases

$$
S_{R}(\lambda, A, x)=B(n) \int_{E(u)} S_{R}(\lambda, I, x-u) f(u) d u
$$

and the boundedness of the set $\left\{S_{R}(\lambda, A, 0)\right\}$ implies (10).

(D) $(10) \rightarrow \lambda \in(M, M),(10) \rightarrow \lambda \in(C, C)$, and $(10) \rightarrow \lambda \in(L, L)$.

From (5) and (10) we have

${ }^{4} \mathrm{~S}$. Banach, Théorie des opérations lınéaires, Monografje Matematyczne, vol. 1, Warsaw, 1932, p. 80.

5 The notation $\int_{E(u)} f(x, u) d \Phi(E)$ is used to indicate the integral over $E$ of $f$ regarded as a function of a point $(u)$. 


$$
\begin{aligned}
S_{R}(\lambda, A, x) & =\sum_{(k)} \Psi\left(\frac{|K|}{R}\right) A(k)(2 \pi)^{-n} \int_{E(u)} e^{-i(k \cdot x-u)} d \Phi(E) \\
& =(2 \pi)^{-n} \int_{E(u)} S_{R}(I, A, x-u) d \Phi(E),
\end{aligned}
$$

and the boundedness, uniform convergence, or mean convergence of $\left\{S_{R}(I, A, x)\right\}$ implies the same for $\left\{S_{R}(\lambda, A, x)\right\}$.

(E) $\lambda \in(L, L) \rightarrow(10)$.

Suppose (10) does not hold. Then there is a sequence $\left\{R_{m}\right\}$ and a sequence of sets $\left\{G_{m}\right\}$, each $G$ being a finite sum of nonoverlapping cubes of $E$, such that

$$
\int_{G_{m}} S_{R_{m}}(\lambda, A, x) d x=(2 \pi)^{-n} \int_{E(u)} f(u)\left\{\int_{G_{m}} S_{R_{m}}(\lambda, I, x-u) d x\right\} d u
$$

is not bounded. But this is a sequence of linear functionals defined over $L$, of norm

$$
\max _{u}\left|\int_{G_{m}} S_{R_{m}}(\lambda, I, x-u) d x\right|,
$$

and by the theorem of Banach and Steinhaus ${ }^{4}$ there is an $f \in L$ such that

$$
\left\{\int_{E} S_{R_{m}}(\lambda, A, x) d x\right\}
$$

is unbounded, and hence $\subseteq(\lambda, A, x) \notin S$, which obviously implies $\Im(\lambda, A, x) \notin L$, contrary to hypothesis.

THEOREM 2. The transformation classes $(S, L)$ and $(M, C)$ are identical, and $\lambda$ belongs to each of them if and only if $\subseteq(\lambda, I, x) \in L$, or

$$
\lim _{R, R^{\prime} \rightarrow \infty} \int_{E}\left|S_{R}(\lambda, I, x)-S_{R^{\prime}}(\lambda, I, x)\right| d x=0 .
$$

Proof. (A) $\lambda \in(S, L) \rightarrow(11)$, immediately, from Lemma 1 .

(B) $(11) \rightarrow \lambda \in(S, L)$.

Since $S_{R}(\lambda, A, x)=(2 \pi)^{-n} \int_{E(u)} S_{R}(\lambda, I, x-u) d \Phi(E)$ it follows that (11) implies the convergence in the mean of $\left\{S_{R}(\lambda, A, x)\right\}$.

(C) $(11) \rightarrow \lambda \in(M, C)$.

Since $S_{R}(\lambda, A, x)=(2 \pi)^{-n} \int_{E(u)} S_{R}(\lambda, I, x-u) f(u) d u$, with $|f| \leqq M$ by hypotheses, the uniform convergence of the sequence $\left\{S_{R}(\lambda, A, x)\right\}$ follows immediately from (11).

(D) $\lambda \in(M, C) \rightarrow(11)$. 
Writing

$$
S_{R}(\lambda, A, x)=(2 \pi)^{-n} \int_{E(u)} S_{R}(\lambda, I, x-u) f(u) d u,
$$

we have

$$
S_{R}(\lambda, A, 0)=(2 \pi)^{-n} \int_{E} S_{R}(\lambda, I, u) f(u) d u=U_{f}\left(S_{R}(\lambda, I, u)\right)
$$

which defines a linear function $U_{f}$. The sequence of values $\left\{U_{f}\left(S_{R}(\lambda, I, u)\right)\right\}$ converges for every bounded $f$, that is, $\left\{S_{R}(\lambda, I, u)\right\}$ converges weakly. But $L$ is weakly complete, ${ }^{6}$ whence there is an $F \in L$ such that

$$
\lim _{R \rightarrow \infty} \int_{E}\left\{S_{R}(\lambda, I, u)-F(u)\right\} f(u) d u=0
$$

for every bounded $f$. Consider now the two series

$$
\Im(F, u) \simeq \sum_{(k)} C(k) e^{i(k \cdot u)}
$$

and

$$
\Im(\lambda, I, u) \simeq \sum_{(k)} \lambda(k) e^{i(k \cdot u)}
$$

and note that

Let

$$
S_{R}(\lambda, I, u)=\sum_{(k)} \Psi\left(\frac{|K|}{R}\right) \lambda(k) e^{i(k \cdot u)}
$$

$$
U_{N}(g)=\int_{E} g(u) e^{-i(N \cdot u)} d u,
$$

which is linear on $L$. Now

whence

$$
\begin{aligned}
\lim _{R \rightarrow \infty} U_{N}\left(S_{R}(\lambda, I, u)\right) & =\lim _{R \rightarrow \infty} \Psi\left(\frac{|N|}{R}\right) \lambda(N)=\lambda(N) \\
& =U_{N}(F)=C(N)
\end{aligned}
$$

$$
S_{R}(\lambda, I, u)=S_{R}(F, u)
$$

But

- S. Banach, Théorie des opérations linéaires, Monografje Matematyczne, vol. 1, Warsaw, 1932, p. 141. 


$$
\lim _{R \rightarrow \infty} \int_{E}\left|S_{R}(F, u)-F(u)\right| d u=0
$$

which, in view of the preceding equality, implies (11).

THEOREM 3. The transformation classes $(S, M),(L, C)$, and $(L, M)$ are identical, and $\lambda$ belongs to each of them if and only if $\subseteq(\lambda, I, x) \in M$, or

$$
\left|S_{R}(\lambda, I, x)\right| \leqq M
$$

Proof. (A) $\lambda \in(S, M) \rightarrow(12)$ follows immediately from Lemma 1. (B) $(12) \rightarrow \lambda \in(S, M)$.

Since

$$
S_{R}(\lambda, A, x)=\int_{E(u)} S_{R}(\lambda, I, x-u) d \Phi(E),
$$

we have from (12)

$$
\left|S_{R}(\lambda, A, x)\right| \leqq M \int_{E}|d \Phi(E)|, \quad \text { and } \quad \Im(\lambda, A, x) \in M .
$$

(C) (12) $\rightarrow \lambda \in(L, C)$.

We have immediately

$$
\left|S_{R}(\lambda, A, x)-S_{R^{\prime}}(\lambda, A, x)\right| \leqq M \int_{E}\left|S_{R}(I, A, x)-S_{R^{\prime}}(I, A, x)\right| d x
$$

where $\Im(\lambda, I, x)=\Im(f, x)$, and $|f| \leqq M$. The integral on the right approaches 0 as $R$ and $R^{\prime}$ approach infinity, whence $\Im(\lambda, A, x) \in C$.

(D) $\lambda \in(L, C) \rightarrow(12)$.

We write immediately

$$
(2 \pi){ }^{n} S_{R}(\lambda, A, x)=\int_{E(u)} S_{R}(\lambda, I, x-u) f(u) d u
$$

and (12) follows from a theorem of Steinhaus and Banach. ${ }^{4}$

(E) $\lambda \in(L, C) \rightarrow \lambda \in(L, M)$ is obvious.

(F) $\lambda \in(L, M) \rightarrow \lambda \in(L, C)$.

Since

$$
S_{R}(\lambda, A, x)=(2 \pi)^{-n} \int_{E(u)} S_{R}(\lambda, I, x-u) f(u) d u
$$

and $\left|S_{R}(\lambda, A, x)\right| \leqq M(f)$ by hypothesis, it follows that $S_{R}(\lambda, A, x)$ exists for every $f \in L$, and $\left\{S_{R}(\lambda, A, 0)\right\}$ is a sequence of linear functionals on $L$, whose norms are 


$$
\text { ess } \sup _{u}\left|S_{R}(\lambda, I, u)\right|=\max _{u}\left|S_{R}(\lambda, I, u)\right| \text {, }
$$

and for every $f \in L$

$$
\left|S_{R}(\lambda, A, 0)\right| \leqq M(f),
$$

whence, by the theorem of Steinhaus and Banach ${ }^{4}$ the norms are uniformly bounded. Hence $\lambda \in(L, M) \rightarrow(12) \rightarrow \lambda \in(L, C)$.

THEOREM 4 . $\lambda$ belongs to the transformation class $(S, C)$ if and only if $\Im(\lambda, I, x) \in C$, or

$$
\lim _{R, R \rightarrow \infty}\left|S_{R}(\lambda, I, x)-S_{R^{\prime}}(\lambda, I, x)\right|=0 \text {, uniformly in } x \in E .
$$

Proof. (A) $\lambda \in(S, C) \rightarrow(13)$ immediately from Lemma 1.

(B) $(13) \rightarrow \lambda \in(S, C)$.

Since

$$
\begin{aligned}
S_{R}(\lambda, A, x)-S_{R^{\prime}}(\lambda, A, x)= & (2 \pi)^{-n} \int_{E(u)} S_{R}(\lambda, I, x-u) d \Phi(E) \\
& -(2 \pi)^{-n} \int_{E(u)} S_{R^{\prime}}(\lambda, I, x-u) d \Phi(E),
\end{aligned}
$$

we may write

$$
\begin{aligned}
\left|S_{R}(\lambda, A, x)-S_{R^{\prime}}(\lambda, A, x)\right| & \\
& \leqq \sup _{x \in E}\left|S_{R}(\lambda, I, x)-S_{R^{\prime}}(\lambda, I, x)\right| \int_{E}|d \Phi(E)|
\end{aligned}
$$

and the uniform convergence of $\left\{S_{R}(\lambda, A, x)\right\}$ follows from that of $\left\{S_{R}(\lambda, I, x)\right\}$.

6. Conclusion. Of the factor sequence transformations among the classes $S, L, M$, and $C$, all of those characterizable in terms of these classes applied to $\Im(\lambda, I, x)$ have been discussed. The class $(L, M)$ of transformations does not exist in a proper sense since, by Theorem 3 , its range is a subset of $C$ contained in $M$. The results of the paper may be taulated as follows:

$$
\begin{aligned}
& \Im(\lambda, I, x) \in S \equiv \lambda \in(S, S) \equiv(L, L) \equiv(M, M) \equiv(C, C) \\
& \Im(\lambda, I, x) \in L \equiv \lambda \in(S, L) \equiv(M, C) \\
& \Im(\lambda, I, x) \in M \equiv \lambda \in(S, M) \equiv(L, C) \\
& \Im(\lambda, I, x) \in C \equiv \lambda \in(S, C)
\end{aligned}
$$

Brown University and The Citadel 